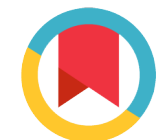

Check for updates

\title{
Development of an early-stage femoral head necrosis rabbit model using methylprednisolone and Complete Freund's Adjuvant
}

\author{
Lan Thi Phi,2, Ha Thi -Ngan Le 1,2, Thuy Thi -Thanh Dao1,2, \\ Khanh Dinh-Van Nguyen2, Khanh Hong-Thien Bui'3, \\ Phuong Thi-Bich Le4, Ngoc Bich Vu1,2, Phuc Van Pham 1,2,*
}

*For correspondence:

pvphuc@hcmus.edu.vn

Competing interests: The authors declare that no competing interests exist.

Received: 20 September 2017 Accepted: 20 November 2017 Published: 27 November 2017

Copyright The Author(s) 2017. This article is published with open access by BioMedPress.

This article is distributed under the terms of the Creative Commons Attribution License (CC-BY 4.0) which permits any use, distribution, and reproduction in any medium, provided the original author(s) and the source are credited.

\section{Abstract}

Introduction: Pathological animal models provide the foundation for developing new methods for disease treatment. This research aims to establish a rabbit model of femoral head necrosis. Methods: Osteonecrosis of the femoral head (ONFH) was induced in rabbits by using methylprednisolone (MPS) combined with Complete Freund's Adjuvant (CFA). New Zealand White rabbits were divided into two groups. ONFH group $(n=10)$ was given an intramuscular injection of $0.5 \mathrm{mg} / \mathrm{kg}$ CFA and $40 \mathrm{mg} / \mathrm{kg}$ methylprednisolone. Normal group $(n=6)$ received normal saline at the same location and same volume as those in ONFH group. The efficiency of the ONFH rabbit model was assessed at week 7 after the last injection. Body weight was detected, and the histological structure of head femoral and bone were assessed by H\&E staining. The empty lacunae were counted. Cartilage degeneration was evaluated using image analysis software. Blood vessel density was assessed after ink artery infusion. The cell cycle of bone marrow-derived mononuclear cells was analyzed by flow cytometry. Results: The results showed that there was no difference in body weight changes of rabbits between the two groups. However, the bone morphology and cartilage surface of the femoral head showed abnormalities in the ONFH group. The percentage of empty osteocyte lacunae was significantly higher in ONFH group than normal group. 
Chondrocyte degeneration and fibrocartilage expression were observed in the ONFH group. Compared to the normal group, the ONFH group had less ink-stained blood vessels. However, the fraction of bone marrow-derived mononuclear cells in $\mathrm{S}$ phase and G2/M phase of the cell cycle was significantly increased in the ONFH group. Conclusion: Thus, CFA combined with MPS for 7 weeks can be used to establish an early-stage femoral head necrosis model in rabbits.

\section{Keywords}

Complete Freund's Adjuvant, Methylprednisolone, Osteonecrosis of the femoral head, Rabbit model

\section{Introduction}

Osteonecrosis of the femoral head is defined as the death of bone and bone marrow cells. As a result, blood supply is disrupted and the femoral head is destroyed, leading to an increase in intraosseous pressure. The main causes include alcohol, trauma, steroid-based medicine for autoimmune diseases, and chronic inflammation (Mont and Hungerford, 1995). There is a relationship between glucocorticoid and bone death, which appear due to blood stasis and ischemia in the trabecular bone, and in autoimmune diseases. Increase of osteocyte apoptosis owing to micro damage in the bone could have a role too (Mendiratta et al., 2008). The most common risk factors for osteonecrosis are the use of corticosteroids, especially in high doses (independent variable). Studies suggest that long-term use of oral or intravenous (IV) corticosteroids is associated with non-traumatic osteonecrosis (Yamamoto et al., 1995; Yamamoto et al., 1997).

Although the disease can be treated by conservation or surgical methods, the results are not as expected. This causes great inconvenience to the patient. Early diagnosis of the disease and appropriate treatment are important to protect the integrity of the femoral head. It is important to establish an animal ONFH model with high reproducibility of necrosis. Furthermore, it is important to deepen the understanding of the prevention, diagnosis and treatment of steroid-induced ONFH.

Many studies have reported that the use of glucocorticoid alone or in combination with certain substances, such as LPS or horse serum, can induce early avascular necrosis of the femoral head (Li et al., 2004; Wen et al., 2008a). CFA is widely used in animal models of osteoarthritis or autoimmune diseases (Hirose and Tanaka, 2011; McCarson, 2015). Freund's supplement is an emulsified solution in mineral oil and used as an agent to stimulate immune 
responses and enhance the production of pro-inflammatory elements (e.g. cytokines IL-1 $\beta$, TNF- $\alpha$ and IL-6).

The aim of this study was to establish an experimental model of ONFH in rabbits induced by a combination of MPS and CFA.

\section{Materials-Methods}

\section{Experimental animals}

All experimental rabbits were fed in a single cage and received a standard laboratory diet. Experimental protocols and animal care methods were approved by the Animal Experimentation Ethics Committee of the Stem Cell Institute, University of Science, Vietnam National University, Ho Chi Minh City, Vietnam.

\section{Establishment of a rabbit model of ONFH}

Male New Zealand white rabbits (28 weeks of age), weighing 2.5-3.5 kg, were separated into two groups. The ONFH group $(n=10)$ was injected in the gluteus medius site with a dose of $0.5 \mathrm{mg} / \mathrm{kg}$ CFA (Santa Cruz, USA) at day 1 and $40 \mathrm{mg} /$ $\mathrm{kg}$ MPS (Pfizer, New York city, NY) at day 7. The normal group $(n=6)$ received $0.9 \%$ saline at the same location and volume as those in the ONFH group. Rabbits were assessed at week 7 after the last injection. All rabbits were followed to record any body weight changes.

\section{Morphological and histopathological assays}

Seven weeks later, rabbits were sacrificed. Femoral head and thighbone were collected. The morphology was observed and recorded using a camera.

In the next step, both femoral head and thighbone were fixed in 10\% paraformaldehyde for 3 days. After that they were soaked in 10\% disodium ethylene diamine triacetic acid (Na2-EDTA) to decalcify. Na2-EDTA solution was refreshed every 2 days until sufficient demineralization was achieved. Demineralization was detected by stabbing a needle to the bone and observing the surface color change of samples. The samples were then dehydrated in $30 \%$ sucrose solution. After 2 days, the samples were cut into 7-10 $\mu$ m-thick sections and stained with hematoxylin-eosin and safranin $O$. The changes in the histological structure of the periosteum, trabeculae and cartilage were observed using a microscope. Ten random fields were chosen to count the number of empty osteocyte lacunae. Bone damage level was detected by the rate of empty osteocyte lacunae. 


\section{Ink artery infusion angiography}

Ink artery infusion angiography was conducted according to the method of Wen Qian et al. (Wen et al., 2008b). Briefly, the rabbit was anesthetized and injected with heparin $(5,000 \mathrm{IU} / \mathrm{mL})$ into an ear vein. The abdomen was operated to expose the abdominal aorta and the abdominal vein was cut. A 12-gauge syringe was inserted in the opposite direction of the heart. About 2,000 mL of physiological saline solution was pumped to wash away the blood from the body. Then, about $100 \mathrm{~mL}$ of gelatin and ink mixture (at a rate of 7:3 in phosphate-buffered saline) were injected continuously until the skin and nails became uniformly black. Viscera from the rabbit was removed and kept at $4{ }^{\circ} \mathrm{C}$ overnight. Over the next day, the femur was collected and fixed in $10 \%$ paraformaldehyde for 3 days. Subsequently, samples were decalcified as described above and cut into $30 \mu \mathrm{m}$-thick sections and blood vessel density was observed using a microscope.

\section{Cell Cycle}

To assess the effect of CFA and MPS on the proliferation and apoptosis of nuclear cells in bone marrow, rabbits received epidural anesthesia using $3 \%$ Novocain (Hanvet, Ho Chi Minh city, Vietnam). The bone marrow was harvested through a hole in the top of the ilium with a 18-gauge biopsy needle. Bone marrow-derived nuclear cells were enriched by incubation in hemolysis solution. Nuclear cells were fixed in $70 \%$ ethanol for 24 hours and stained with propidium iodide (Sigma-Aldrich, Louis St, MO) for 10 minutes. Cell cycle was analyzed by flow cytometry.

\section{Statistical Analysis}

All data were analyzed using Graphpad Prism 6.0 software. Images were analyzed using ImageJ software.

\section{Results}

\section{Morphological changes of the cartilage and bone}

After 7 weeks of drug administration, bone morphology had significant changes in normal rabbits compared to treated rabbits (Fig. 1). The femoral head was shiny, smooth and opalescent in the normal group. In contrast, in the treatment group, the cartilage surface of the femoral head was slightly rough, uneven and pink.

\section{Osteocyte damage}

In group B, almost all osteocyte lacunae were filled with cells that was identified by the presence of oval-shaped nuclei inside (Fig. 2B). In contrast, the number 
of empty lacunae was displayed at many bone sites in the ONFH group. The percentage of empty osteocyte lacunae was significantly higher in ONFH group than normal group $(p<0.05)$. The percentage of empty bone lacunae defects in the normal group was $0.06 \pm 0.01 \%$, while for the ONFH group it was $0.17 \pm$ $0.02 \%$ (Fig. 2).
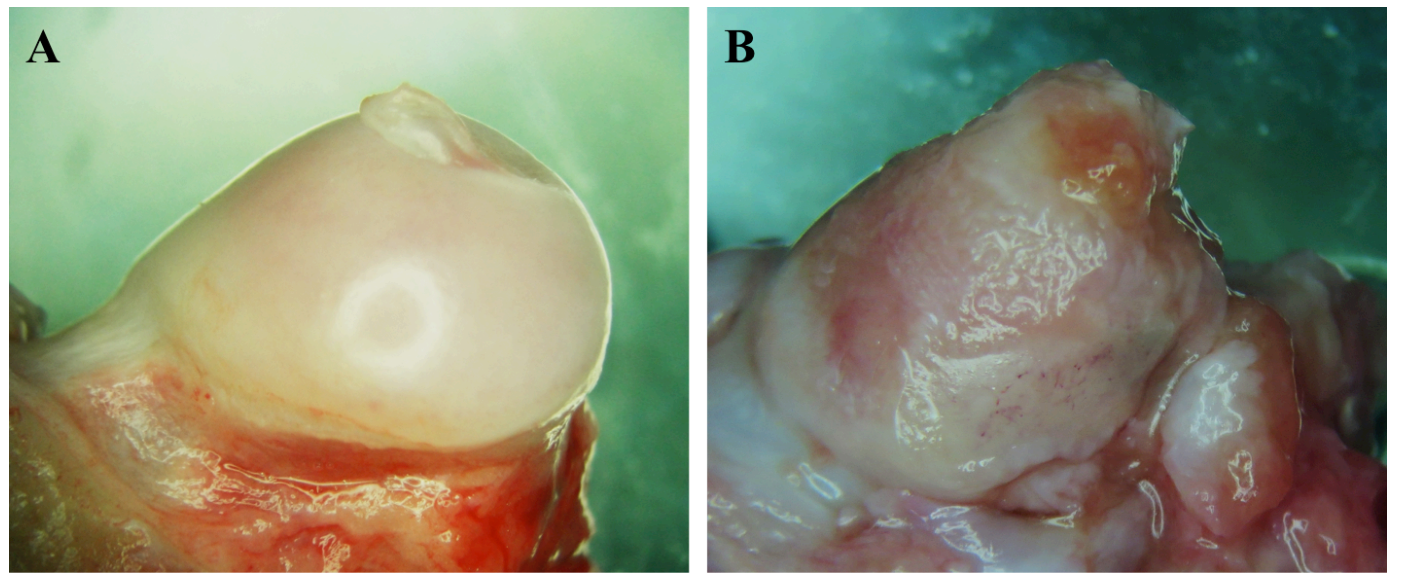

Figure 1. The morphological changes of the femoral head. (A) Normal groupfemoral head was shiny, smooth and opalescent; (B) ONFH group- femoral head was slightly rough, uneven and pink after 7 weeks.
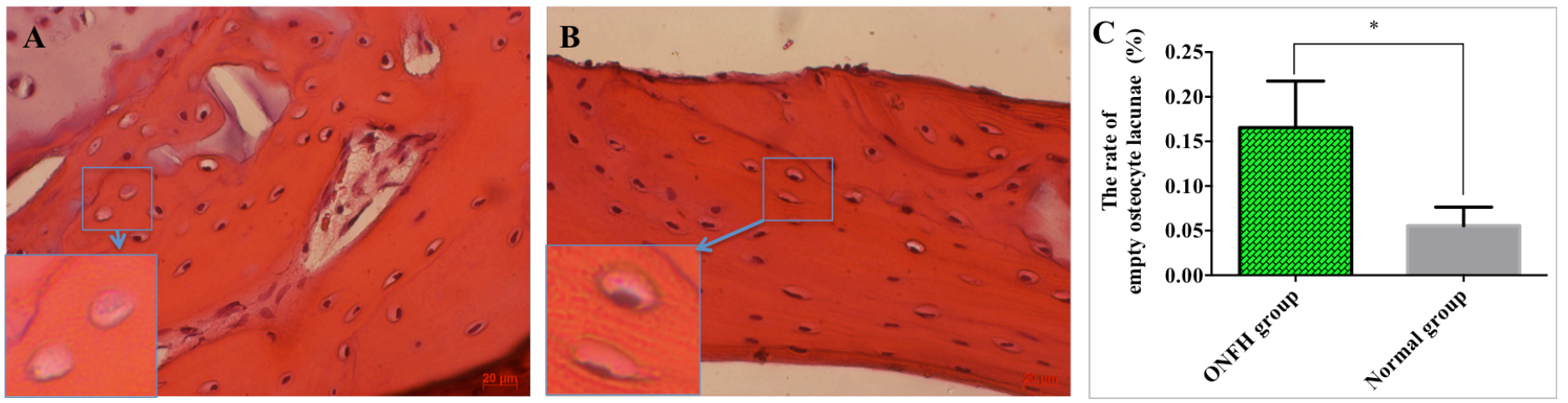

Figure 2. Histology of the bone (H\&E staining). (A) The number of empty osteocyte lacunae was displayed at many bone sites in the ONFH group; (B) Normal bone showed that there were a few empty lacunae in the normal group; (C) the percentage of empty lacunae in both groups at week 7 .

\section{Proteoglycan content}

The concentration of proteoglycans that displayed by orange color was significant higher in the normal group (Fig. 2A,B), compared to the ONFH group (Fig. 3A,D) at 7 weeks. Statistical data showed that the proteoglycan level of the 
control group was $4.14 \pm 0.29 \mu \mathrm{m}$ while for the ONFH group the values were $2.29 \pm 0.17 \mu \mathrm{m}(\mathrm{p}<0.05)$.

At the zone of calcified and hypertrophic cartilage, the fibrocartilage (characterized by the presentation of collagen via green color) was strongly expressed in the ONFH group after staining with safranin $O$ and fast green (narrow; Fig. 3Di, as opposed to the control group (narrow, Fig. 3B).
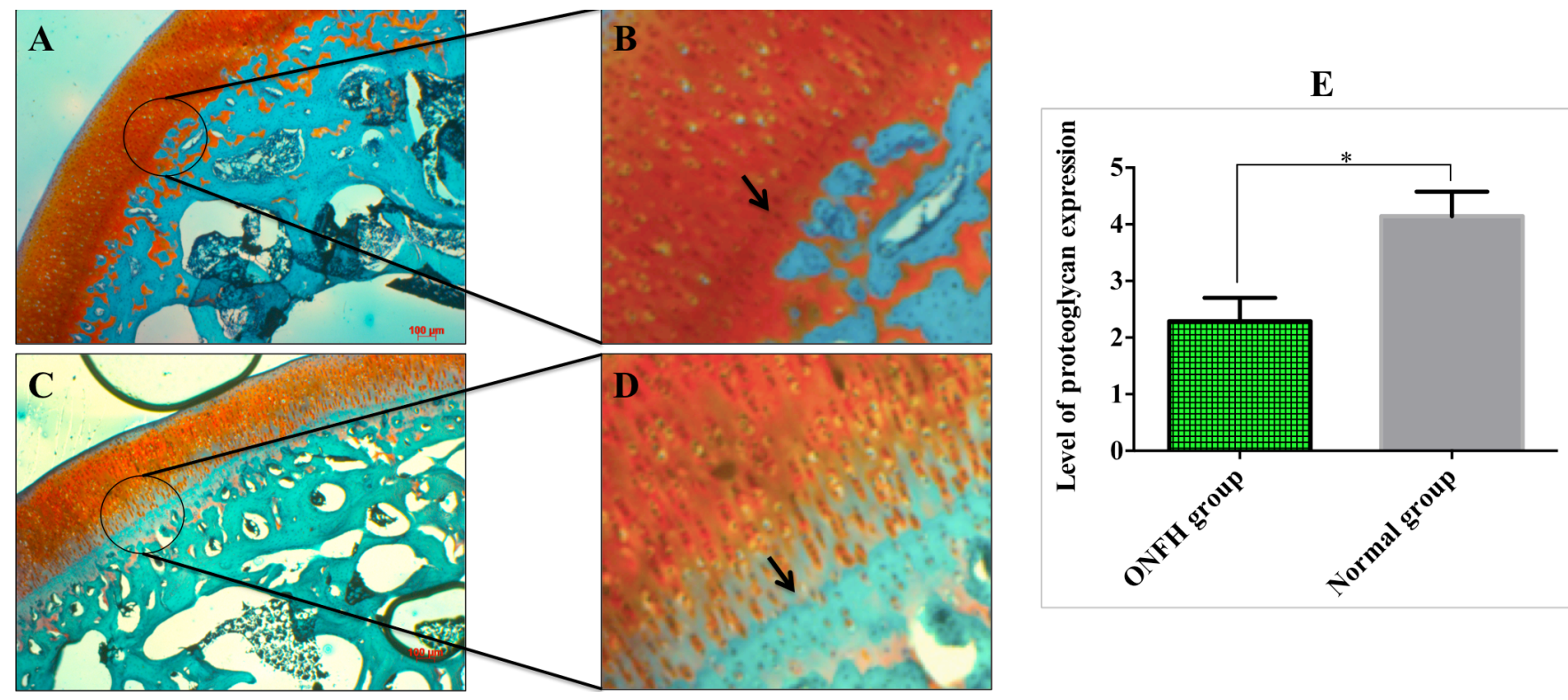

Figure 3. Reduction of proteoglycan expression level at the femur head. Sections from femoral head were stained with safranin $O$ and fast green. The sections showed that more collagen and less proteoglycan was expressed in the ONFH group (C, D) than in the normal group (A, B). The orange-red color was considered to represent proteoglycan while the green color represented collagen. Quantification of proteoglycan levels showed that the amount of proteoglycan in calcified and hypertrophic cartilage in the ONFH group was significantly less than that of the normal group (E).

\section{Ink artery infusion angiography}

The capillaries network in the medullary cavity was high at femoral heads of the normal group, as displayed by the presentation of black color after ink injection into the abdominal aorta (Fig. 4A). In contrast, in the ONFH model group, the density of black medullary cavity was less, indicating that the capillary network was sparse and that there might have been some injuries within the femoral head (Fig. 4B). 

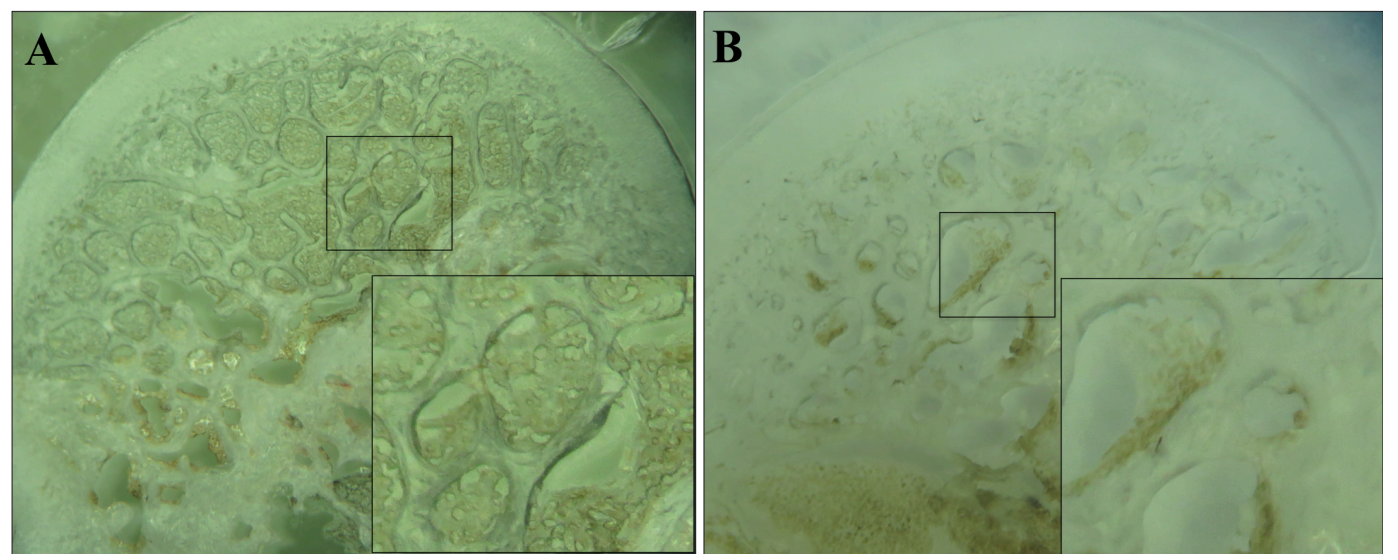

Figure 4. Ink artery infusion angiography of the femoral head. The density of ink-stained capillaries was higher in normal group (A) compared to ONFH model group (B).

\section{Cell cycle}

Compared to the control group, the proportion of bone marrow derived nuclear cells in the ONFH group at G0/G1 phase was significant increased $(p<0.05)$ and $\mathrm{S}$ and $\mathrm{G} 2 / \mathrm{M}$ significantly decreased after week 7 . The rate of apoptotic cells in the ONFH group was higher than for the control group at week 7; however, the difference was not significant (Fig. 5).

\section{Discussion}

Models of femoral head necrosis have been established in a variety of animal species, such as ostriches (Conzemius et al., 2002), dogs (Nakamura et al., 1997) rats (Norman et al., 1998), and rabbits (Yamamoto et al., 1995). Each species has its own strengths and weaknesses in establishing a successful disease model. The success depends, in part, on the laboratory conditions. Both traumatic and non-traumatic osteonecrosis models have been reported in previous studies (Hwang et al., 2011; Le et al., 2016; Mont and Hungerford, 1995). Using drugs to create an animal disease model is a non-traumatic method. This method intends to limit invasiveness and reduce pain in animals. In our study, a combination of endotoxin and corticosteroid was used to enhance the immune response of animals, thereby increasing the effectiveness of establishing a successful animal model.

Damage of osteocytes may lead to their apoptosis or death. As a consequence, the bones get weaker (Heino et al., 2009). A study showed that osteocyte apoptosis was promoted by glucocorticoids (Bonewald, 2011). Cartilage injuries can cause tissue structural deformation. MPS and CFA have been demonstrated 
to induce changes in cartilage structure. Many glycosaminoglycans attach to a core protein to form a proteoglycan, which are secreted into the cartilage matrix by chondrocytes. The interaction between chondrocytes and the cartilage matrix play an important role in proliferation and cartilage remodeling (Esko et al., 2009).
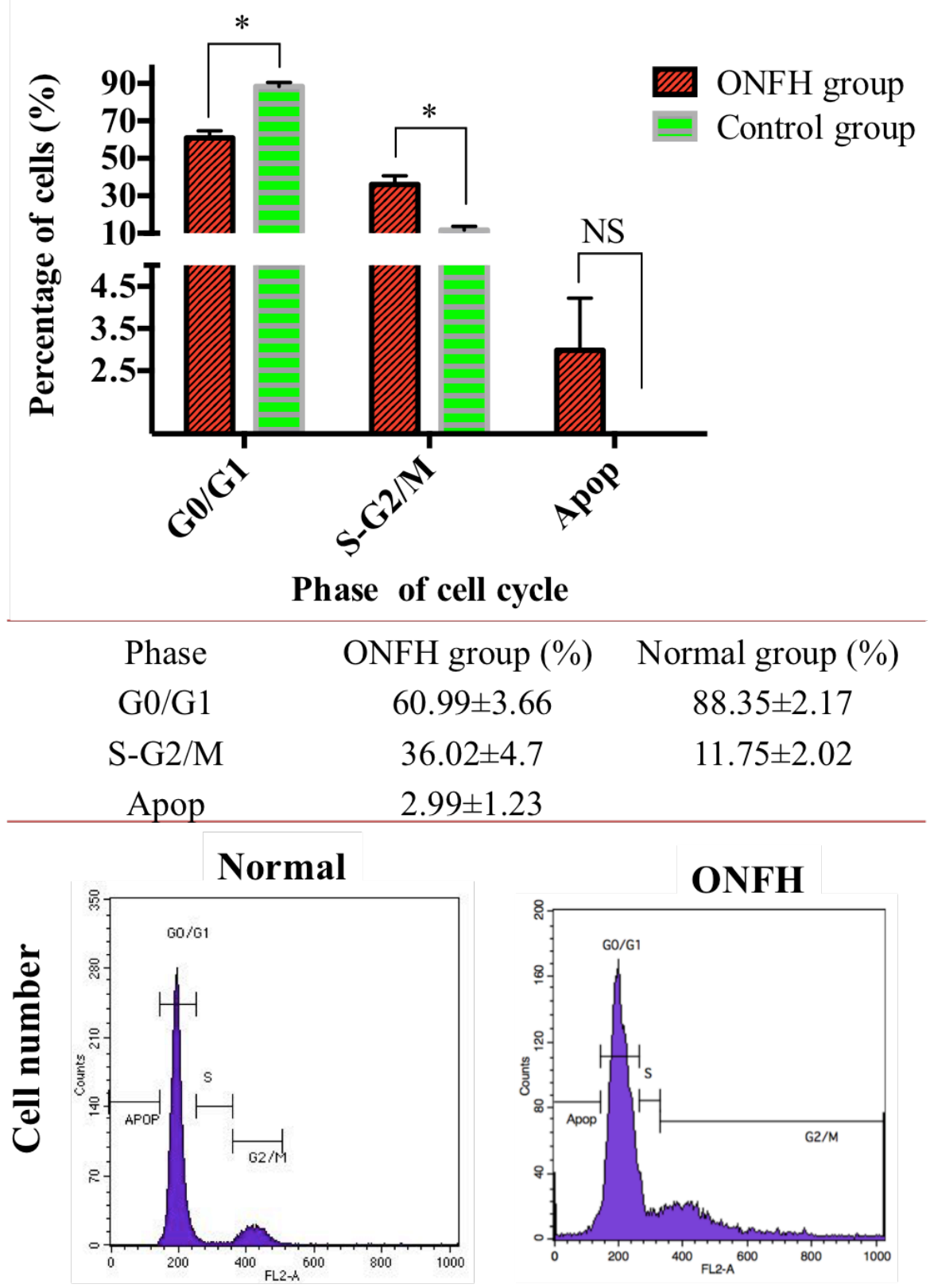

Propidium iodide intensitive

Figure 5. The cell cycle under the influence of methylprednisolone and CFA. The percentage of bone marrow-derived nuclear cells at G0/G1 and S-G2/M phase after 7 weeks of drug induction in the ONFH group was significantly different when compared with the normal group ( $\left.{ }^{*} p<0.05 ; n=5\right)$. 
In our study, the results from safranin O/fast green staining and ink artery perfusion angiography showed that proteoglycans and blood vessels at the femoral head were damaged after methylprednisolone and CFA injection. However, cell cycle analysis showed that at the same time, the percentage of cells at S/G2M phase was higher in the ONFH rabbit group than in the normal rabbits $(p<0.05)$. The rate of increase in cell division may play a role in repairing damaged tissues due to drug administration at an early stage. On the other hand, the percentage of apoptotic cells and empty bone lacunae was greater in the ONFH group than normal group, indicating that MPS combined with CFA had a severe impact on bone.

\title{
Conclusion
}

MPS combined with CFA can be used in rabbits to induce a model of early osteonecrosis of femoral head. However, further studies should be performed to provide more experimental data to support its preclinical application.

\section{Abbreviations}

\author{
CFA Complete Freund's Adjuvant \\ IV Intravenous \\ LPS Lipopolysaccharide \\ MPS Methylprenisolone \\ ONFH Osteonecrosis of the femoral head
}

\section{Acknowledgements}

This work was supported by University of science, Vietnam National University Ho Chi Minh City (VNU-HCM) under grant number T2016-21, and Vietnam National University Ho Chi Minh City (VNU-HCM) under grant number TX2017-18-02.

\section{References}

Bonewald, L. F. (2011). The Amazing Osteocyte. Journal of Bone and Mineral Research, 26(2), 229-238. https://doi.org/10.1002/jbmr.320

Conzemius, M.G., Brown, T.D., Zhang, Y., and Robinson, R.A. (2002). A new animal model of femoral head osteonecrosis: one that progresses to human-like mechanical failure. Journal of orthopaedic research : official publication of the Orthopaedic Research Society 20, 303-309. https://doi.org/10.1016/S0736-0266(01)00108-5 
Esko, J. D., Kimata, K., \& Lindahl, U. (2009). Proteoglycans and Sulfated Glycosaminoglycans. In A. Varki, R. D. Cummings, J. D. Esko, H. H. Freeze, P. Stanley, C. R. Bertozzi, . . Etzler, M. E. (Eds.), Essentials of Glycobiology. Cold Spring Harbor, NY: Cold Spring Harbor Laboratory Press The Consortium of Glycobiology Editors, La Jolla, California.

Heino, T.J., Kurata, K., Higaki, H., and Vaananen, H.K. (2009). Evidence for the role of osteocytes in the initiation of targeted remodeling. Technology and health care : official journal of the European Society for Engineering and Medicine 17, 49-56.

Hirose, J., \& Tanaka, S. (2011). [Animal models for bone and joint disease. CIA, CAIA model]. Clinical Calcium, 21, 253-259.

Hwang, Y., Park, J., Choi, S. H., \& Kim, G. (2011). Traumatic and Non-traumatic Osteonecrosis in the Femoral Head of a Rabbit Model. Laboratory Animal Research, 27(2), 127-131. https://doi.org/10.5625/lar.2011.27.2.127

Le, H. T.-N., Phi, L. T., Dao, T. T.-T., Phan, N. K., Van Pham, P., \& Vu, N. B. (2016). A mouse model of osteonecrotic femoral head induced by methylprednisolone and liposaccharide. Biomedical Research and Therapy, 3(3), 12. https://doi.org/10.7603/ s40730-016-0012-x

Li, Y., Chen, J., Zhang, Z., Wang, K., Tong, Z., and Yan, H. (2004). The experimental study on treatment of glucocorticoid-induced ischemic necrosis of femoral head by gu fu sheng capsule. Journal of traditional Chinese medicine $=$ Chung $i$ tsa chih ying wen pan 24, 303-307.

McCarson, K.E. (2015). Models of Inflammation: Carrageenan- or Complete Freund's Adjuvant (CFA)-Induced Edema and Hypersensitivity in the Rat. Current protocols in pharmacology 70, 5.4.1-9.

Mendiratta, V., Khan, A., \& Solanki, R. S. (2008). Avascular necrosis: A rare complication of steroid therapy for pemphigus. Indian Journal of Dermatology, 53(1), 28-30. https:// doi.org/10.4103/0019-5154.39739

Mont, M. A., \& Hungerford, D. S. (1995). Non-traumatic avascular necrosis of the femoral head. The Journal of Bone and Joint Surgery. American Volume, 77(3), 459-474. https:// doi.org/10.2106/00004623-199503000-00018

Nakamura, T., Matsumoto, T., Nishino, M., Tomita, K., \& Kadoya, M. (1997). Early magnetic resonance imaging and histologic findings in a model of femoral head necrosis. Clinical Orthopaedics and Related Research, 68-72.

Norman, D., Reis, D., Zinman, C., Misselevich, I., \& Boss, J. H. (1998). Vascular deprivation-induced necrosis of the femoral head of the rat. An experimental model of avascular osteonecrosis in the skeletally immature individual or Legg-Perthes disease. International Journal of Experimental Pathology, 79(03), 173-181. https://doi.org/ 10.1046/j.1365-2613.1998.00063.x

Wen, Q., Ma, L., Chen, Y. P., Yang, L., Luo, W., \& Wang, X. N. (2008a). A rabbit model of hormone-induced early avascular necrosis of the femoral head. Biomedical and environmental sciences. BES, 21, 398-403.

Wen, Q., Ma, L., Chen, Y. P., Yang, L., Luo, W., \& Wang, X. N. (2008b). Treatment of avascular necrosis of the femoral head by hepatocyte growth factor-transgenic bone marrow stromal stem cells. Gene Therapy, 15(23), 1523-1535. https://doi.org/10.1038/ gt.2008.110 
Yamamoto, T., Hirano, K., Tsutsui, H., Sugioka, Y., \& Sueishi, K. (1995). Corticosteroid Enhances the Experimental Induction of Osteonecrosis in Rabbits With Shwartzman Reaction. Clinical Orthopaedics and Related Research, (316): 235-243.

Yamamoto, T., Irisa, T., Sugioka, Y., Sueishi, K., Yamamoto, T., Irisa, T., . . Sueishi, K. (1997). Effects of pulse methylprednisolone on bone and marrow tissues: Corticosteroidinduced osteonecrosis in rabbits. Arthritis and Rheumatism, 40(11), 2055-2064. https:// doi.org/10.1002/art.1780401119 